Revue européenne des sciences sociales

European Journal of Social Sciences

XXXVIII-117| 2000

Métaphores et analogies. Schèmes argumentatifs des sciences sociales

\title{
Le carnet de bord d'un historien bourlingueur
}

Le drapeau et l'identité nationale

\section{Ruggiero Romano}

\author{
(2) OpenEdition \\ Journals \\ Édition électronique \\ URL : http://journals.openedition.org/ress/726 \\ DOI : $10.4000 /$ ress. 726 \\ ISSN : $1663-4446$ \\ Éditeur \\ Librairie Droz \\ Édition imprimée \\ Date de publication : 1 février 2000 \\ Pagination : 211-215 \\ ISBN : 2-600-00409-2 \\ ISSN : 0048-8046 \\ Référence électronique \\ Ruggiero Romano, "Le carnet de bord d'un historien bourlingueur », Revue européenne des sciences \\ sociales [En ligne], XXXVIII-117 | 2000, mis en ligne le 17 décembre 2009, consulté le 01 mai 2019. \\ URL : http://journals.openedition.org/ress/726 ; DOI : 10.4000/ress.726
}

(c) Librairie Droz 


\section{Ruggiero ROMANO}

\section{LE CARNET DE BORD D'UN HISTORIEN BOURLINGUEUR Le drapeau et l'identité nationale}

La nation, vous connaissez? Voyons donc, on nous a enseigné depuis belle lurette que la nation, nos nations, sont assez vieilles : elles remontent à la création de l'État-Nation et, avec un peu de bonne volonté, on peut même remonter plus loin dans le temps, par exemple en France jusqu'au couronnement en 987 de Hugues Capet. Si cette dernière proposition est totalement fausse (il ne s'agit que d'une phrase d'occasion écrite par un historien en 1987, à l'occasion d'un millénaire...), la tendance à faire remonter au $\mathrm{XV}^{\mathrm{e}}$ siècle l'idée de nation est elle aussi discutable car - en compagnie d'un véritablement grand historien, Federico Chabod $-{ }^{1}$ on peut soutenir avec des solides arguments que l'idée de nation s'affirme seulement autour de la moitié du XVIII ${ }^{\mathrm{e}}$ siècle, en Suisse (surtout en Suisse) et en Allemagne (et aussi en France). Plus tard, la révolution française achèvera l'ouvrage et alors on passera très vite de l'idée de nation au nationalisme.

Mais je ne veux pas insister sur ce thème ${ }^{2}$ et reprendre des polémiques qui n'ont rien à voir avec ce que j'aimerais raconter ici

Les couleurs du drapeau mexicain, depuis l'indépendance du pays en 1821, sont vert, blanc, rouge. Rien de très particulier. Infiniment plus intéressants sont les symboles qui apparaissent sur la partie blanche: un monticule de terre qui porte un nopal (le cactus de la figue d'Inde) sur lequel se perche - solidement agrippé par ses serres - un aigle tenant dans son rostre un serpent.

Donc, si un drapeau doit représenter l' identité d'un pays, dans le cas mexicain cette fonction n'est pas assurée par les couleurs (qui pourraient se confondre avec celles du drapeau italien), mais par les symboles. C'est eux qui «font» le drapeau et ils sont anciens, très anciens. Le recours à tout ce qui est antique est fondamental dans la constitution de la légitimité d'une nation. Un jeune pays qui veut affirmer son indépendance nationale a tout intérêt à recourir à des symboles dont les origines se perdent dans la nuit des temps. ${ }^{3}$ De même un régime nouveau essaye toujours (ou assez souvent) de se parer des symboles anciens, comme par exemple

L'idea di Nazione, Bari-Roma, Laterza, 1961

J'en ai déjà parlé dans mon Paese Italia, Roma, Donzelli, 1992.

D. Handelman \& L.Shangar-Handelman, Shaping Time: The Choice of National Emblem of Israel; dans E. Ohnuki-Tierney (Ed.), Culture through Time. Anthropological Approaches, Stanford, Stanford University Press, 1990, pp. 193-226 Qu'il me soit permis de renvoyer aussi aux travaux d'un ami disparu, Valerio Valeri, jeune anthropologue italien émigré aux États Unis où il a enseigné à l'Université de Chicago: voir en particulier son essai Constitutive History: Genealogy and Narrative. Legitimation of Hawaian Kingship, publié dans le volume déjà cité de E. OhnukiTierney, pp. 154-164. 
en France le gouvernement de Vichy, entre 1941 et 1945, adopta des signes et symboles gaulois ${ }^{4}$.

Que sont-ils donc les antiques symboles qui apparaissent dans le drapeau mexicain?

Le monticule à l'origine est en réalité une pierre, représentation du cœur arraché à Cópil, le prototype de l'ennemi des Aztèques. De ce cœur jaillit le nopal (la forme de ses fruits rappelle d'ailleurs celle d'un cœur) qui représente l'arbre cosmique. L'aigle n'est que le double du soleil, un soleil qui triomphe de ses ennemis, symbolisés par le serpent. Et l'ensemble de tous ces éléments représente la fondation primordiale.

Les premières représentations iconographiques de ces symboles nous sont attestées dès le $\mathrm{III}^{\mathrm{e}}$ siècle après le Christ. Elles traversent un laps de temps assez considérable et se heurtent à des faits majeurs comme la conquête espagnole et la christianisation qui en découle.

On pourrait croire que, du fait de l'énorme processus de deculturation du monde indigène, ces symboles étaient destinés à disparaître, en tout cas à perdre leur signification. Or, il n'en est rien; ils luttent avec succès contre les symboles nouveaux: dès le $\mathrm{XVI}^{\mathrm{e}}$ siècle l'aigle mexicain s'introduit partout plus ou moins subrepticement: dans telles sculptures de couvents franciscains ou dans les fresques de couvents augustiniens, et jusque dans l' écusson de la ville de México. Ainsi se met en place une véritable guerre des symboles qui voit le triomphe de la tradition indigène, à tel point que les autorités espagnoles sentent bien que cet oiseau rivalise avec succès avec l'aigle à deux têtes de la monarchie espagnole Un dessin de 1666 montre l'aigle espagnol qui chasse de son nid l'aigle mexicain: une réalité ou plutôt un souhait? Car, si le vice-roi Palafox a ordonné en 1642 de supprimer l'écusson avec l'aigle mexicain et décidé aussi de l'arracher de la fontaine qui est devant le palais vice royal, cet ordre n'a pas été exécuté ou - s'il l'a été - son effet a été de courte durée puisque l'aigle apparaît à nouveau sur la fontaine dans une lithographie de 1761. Par la suite on trouvera les symboles indigènes même sur le fronton des bâtiments publics, l'Hôtel de la Monnaie ou la Douane ou l'Académie des Beaux Arts (cette dernière, il est vrai, ajoutera le laurier au nopal...). Mais il y a un autre chemin plus profond et plus important de ce triomphe de l'aigle mexicain: la vierge de Guadeloupe. Dès le milieu du XVII siècle (en 1648, plus exactement) apparaissent des dessins qui la représentent non plus portée par un ange mais debout sur un nopal avec un aigle qui derrière elle déploie ses ailes. Le symbole du nopal est clair, mais celui de l'aigle est encore ambigu car il s'agit d'une bête à deux têtes et donc il a un lien seulement indirect avec l'aigle mexicain, plus direct avec l'héraldique espagnole. Toutefois ce n'est là qu'une première étape car progressivement la vierge apparaît de plus en plus souvent appuyée sur le nopal surmonté par l'aigle avec le serpent dans le rostre. En somme, dès le XVIII ${ }^{\mathrm{e}}$ siècle, la vierge chrétienne véhicule l'ensemble symbolique indigène.

Dans ces conditions il est clair que la recherche d'un symbole de l'identité de la nation mexicaine au moment où le Mexique conquiert son indépendance, est

4 Cf. le bel essai de K. Pomian, Francs et gaulois, dans P. Nora (Ed.), Les lieux de mémoire, Paris, Gallimard, t. III, 1986, particulièrement pp. 43-46. 
assez simple: quoi de mieux - de plus profondément mexicain - que le monticule, le nopal, l'aigle et le serpent? Cet ensemble symbolique joue parfaitement son rôle aujourd'hui encore.

Tout ce que je viens de dire, je l'ai tiré des multiples richesses offertes par un petit livre de Enrique Florescano ${ }^{5}$. La réputation de cet historien n'est plus à faire: il y a trente ans déjà il s'est imposé au niveau international avec son premier livre sur l'histoire des prix au Mexique. Ensuite, sa recherche l'a porté à s'occuper surtout (mais non exclusivement) d'histoire économique de son pays, des épidémies, des sécheresses, du commerce. Et dans tous ces domaines il a laissé une empreinte très ferme.

Il est évident que ces titres de mérite des années passées ne sont pas pas une raison suffisante pour suivre l'auteur dans la démarche de ce petit livre de poche. Je veux dire qu'on peut ne pas lui reconnaître une autorité suffisante pour le suivre dans sa chevauchée à travers les millénaires à la poursuite des symboles d'identification de la nation mexicaine. Mais le fait est que ce livre s'appuie sur des fondations solides car, depuis une dizaine d'années, Enrique Florescano a publié plusieurs gros ouvrages ${ }^{6}$, à l'érudition impressionnante, sur des thèmes archéologiques, anthropologiques, sociologiques. Ne nous y trompons pas cependant: le problème fondamental de ces dernières recherches n'est ni anthropologique, ni archéologique, ni religieux, il est essentiellement historique: comment se sont formés dans un passé lointain -à la limite de la préhistoire- certains mythes, certaines croyances, et comment se sont-ils conservés ou ont évolué dans la longue durée, jusqu'à nos jours?

Il faut ajouter que Enrique Florescano ne nous a pas offert seulement le fruit de son travail personnel mais qu'il a poursuivi ses recherches sur l'identité nationale dans d'autres livres dont il est le promoteur (l'Editor, comme on dit maintenant) et qui portent tous sa marque ${ }^{7}$, qui reprennent certains des thèmes archéologiques, anthropologiques, sociologiques déjà traités par lui, mais en élargissant considérablement le champ d'investigation.

Je ne veux pas dire que la petite histoire du drapeau écrite récemment par Enrique Florescano serait l'aboutissement, la summa, de ses recherches passées et que celles-ci y «confluent». Plus simplement, je soutiens que ceux qui seraient tentés de considérer «léger» ce petit livre se tromperaient lourdement. Ce drapeau est solidement planté au sommet d'une masse documentaire sans faille. Et c'est cet ensemble qu'il faut prendre en examen. La thèse profonde de Enrique Florescano est que l'idée que dans l'histoire de son pays il y a un avant et un après la conquête espagnole, est fausse ou du moins à fortement nuancer. C'est une idée espagnole, eurocentrique, qui ne correspond pas à la réalité de la conscience indigène (c'est à dire de la masse de la population). Des mythes millénaires se sont

5 La bandera mexicana. Breve historia de su formación y simbolismo, México, F.C.E., 1998

6 Memoria mexicana. Ensayo sobre la reconstrucción del pasado: época prehispanica-1821, México, Editorial Joaquín Mortiz, 1987 (nouvelle édition ampliée, México, F.C.E., 1994); El nuevo pasado méxicano, México, Cal y arena, 1991; El mito de Quetzalcóatl, México, F. C. E., 1993 (trad. angl., Baltimore, John Hopkins University, 1999); Etnia, Estado y Nación. Ensayo sobre las identidades colectivas en México, México, Aguilar, 1997.

7 El patrimonio cultural de México, México, F.C.E., 1993 (nouvelle édition, México, F.C.E., 1997); Mitos mexicanos, México, Aguilar, 1995. 
conservés, tout en se modifiant, et dans ces mythes les mexicains se sont constamment reconnus. Prenons Quetzalcóatl, le serpent à plumes: véritable mythe agricole, héros culturel, il persistera dans la mémoire de la population indigène même pendant la période coloniale, sous la forme d'un apôtre qui serait arrivé au Mexique longtemps avant les espagnols. Autre forme possible de survie, Quetzalcóatl serait un roi-prêtre détrôné qui aurait annoncé l'arrivée des blancs barbus. Mais c'est à la Vierge de Guadeloupe que revient le rôle le plus important dans ce processus de syncrétisme, non seulement parce qu'elle réunit le nopal, l'aigle, le serpent mais aussi parce que, en elle et par elle, survit dans la croyance indigène le souvenir de Tonantzin, la mère antique de tous les dieux.

J'avoue avoir été fasciné par les travaux de Enrique Florescano. Certes, on peut très bien ne pas le suivre dans son idée d'une persistance continue de l'identité «nationale» mexicaine au travers des siècles ${ }^{8}$, mais on ne peut pas ne pas accepter son effort de suivre avec une si grande passion les apparitions et les résurgences de mythes, d'idées par des cheminements compliqués, confus, labyrinthiques. Cette quête reflète certainement son amour de la patrie mais aussi et surtout sa passion d'historien, qui veut se confronter aux problèmes de la longue durée.

Je ne sais pas si Enrique Florescano a raison ou tort, mais de toute façon il me semble que le mérite de son travail de plus de dix ans ne réside pas dans son tort ou sa raison, mais dans l' effort qu'il a accompli. Je veux dire que je ne sais pas, par exemple, si on peut comparer la persistance millénaire de l'ensemble mexicain tel qu'il a été montré et démontré par Florescano au rôle de l' étoile de David dans l'histoire du peuple juif, car il est facile d'objecter qu' il y a au moins deux grosses différences entre les deux exemples:

a) le peuple juif constitue un ensemble dès l'antiquité, avant même la diaspora. Il est difficile de dire que les ethnies qui habitaient l'espace actuel du Mexique aient jamais constitué une unité dans l'antiquité. Je veux bien que sous des formes différentes Quetzalcoatl ait été présent dans toute l'Amérique Centrale, mais cela ne me paraît pas suffisant car nous savons que ces héros culturels, ces héros fondateurs, constituent un élément fondateur d'innombrables sociétés;

b) dans le cas mexicain, il n'y a jamais eu de dispersion du peuple mexicain (et y a-t-il un peuple «mexicain », qui couvre toutes le ethnies, du Nord du pays à la frontière avec le Guatemala?) Au contraire, la millénaire cohésion juive est due en bonne partie à la dispersion, à la nécessité de se conserver dans sa spécificité. Enrique Florescano sait mieux que moi que le projet «impérial» espagnol de créer la «république des espagnols» nettement séparée de la «république des indiens », ne se réalisa jamais et que l'on assista au triomphe du métissage physique (et culturel plus encore).

Cela dit, on ne peut pas balayer d'un geste de la main les résultats des travaux d'Enrique Florescano car - je le répète - ils posent, sur des bases très solides, un problème historiographique très important: celui de la longue durée

8 D'ailleurs, lui même reconnaît qu'il y a des moments de «pulverización » de la mémoire ethnique avec refuge de celle-ci dans une simple mémoire locale. 
Moi aussi je crois avec Enrique Florescano que le syncrétisme entre valeurs indigènes et valeurs hispano-chrétiennes qui se réalisa au Mexique pendant la période coloniale se fit en bonne partie à l'avantage des éléments traditionnels indigènes. Mais je crois aussi que ce même phénomène peut être observé dans le monde andin ${ }^{9}$, sans que dans ce dernier on puisse constater la persistance d'une conscience nationale des anciens symboles jusqu'à nos jours.

Mais alors le problème devient le suivant: pourquoi l'ensemble de symboles si savamment reconstitué par Enrique Florescano joue-t-il encore dans le cas mexicain et pas dans le cas péruvien ou bolivien? Car s'il est vrai que la Vierge Marie véhicule encore aujourd'hui au Pérou l'idée prehispanique de la pacha-mama (la terre mère), il est impossible de dire qu'elle joue dans les consciences péruviennes ou boliviennes le même rôle que la Vierge de Guadeloupe au Mexique. De même, si au Pérou Saint Jacques représente encore le seigneur des foudres prehispanique, il est impossible de lui attribuer la même fonction que Queztalcoatl au Mexique. Inti lui même -le soleil- ne me semble pas jouer un rôle majeur dans la prise de conscience d'identité nationale au Pérou.

En acceptant donc l'idée d'une grande persistance, d'une très longue durée des symboles mexicains mais aussi des andins, reste à savoir pourquoi les uns sont fortement actifs, tandis que les autres ne semblent plus constituer un élément d'agrégation nationale. Faudra-t-il mettre en cause le rôle de l' État et voir en lui l'agent qui a été capable - après l'indépendance de 1821 et surtout après la révolution de 1910 - de susciter au Mexique une conscience nationale en intégrant dans le corps de la nation la population indigène? On pourrait le croire, en lisant en contrepoint par exemple les pages de Enrique Florescano ${ }^{10}$ consacrées au rôle joué par la génération de la «Reforma» (entre 1867 et 1890) et les pages si belles de Alberto Flores Galindo et Manuel Burga dédiées aux hommes de l'oligarchie péruvienne $^{11}$.

De toute évidence, ce problème du rôle de l'État dans la formation de la conscience nationale n'est pas seulement mexicain ou andin, il se pose aussi pour d'autres structures nationales ${ }^{12}$. Et c'est le grand mérite de ces travaux d'Enrique Florescano rapidement présentés ici que de soulever des problèmes qui vont largement au delà du cas mexicain et qui peuvent (devraient) intéresser tous ceux qui aujourd'hui sont attentifs aux problèmes de la nation.

\section{Ecole des hautes études des sciences sociales}

Paris

9 Voir à ce sujet surtout les livres fondamentaux T. Gisbert, Iconografia y mitos indigenas en el arte, La Paz, Gisbert y C.ia Libreros Editores, 1980; A. Flores Galindo, Buscando un Inca, Lima, Editoriaal Horizonte, 1988 e M. Burga, Nacimiento de una utopía. Muerte y resurreción de los Incas, Lima, Instituto de Apoyo Agrario, 1988.

10 Etnia, Estado, Nación..., cit., pp. 431 ss.

11 V. A. Flores Galindo, Aristocracia y plebe, Lima, Mosca Azul, 1984 et M. Burga \& A. Flores Galindo, Apogeo y crisis de la republica aristocratica, Lima, Ediciones «Rikchai Peru», 1979.

12 On peut se demander, par exemple, pourquoi l'idée de Mussolini de ressusciter le mythe de la louve romaine n'eut pas de succès. 